\begin{tabular}{|l|l|l|}
\hline \multicolumn{2}{|c|}{ PublisherInfo } \\
\hline \hline PublisherName & $:$ & BioMed Central \\
\hline \hline PublisherLocation & $:$ & London \\
\hline \hline PublisherImprintName & $:$ & BioMed Central \\
\hline \hline
\end{tabular}

\title{
Micrometastases and survival in women with primary breast
} cancer

\begin{tabular}{|l|l|l||}
\hline \multicolumn{2}{|c||}{ ArticleInfo } \\
\hline \hline ArticleID & $:$ & 3616 \\
\hline \hline ArticleDOI & $:$ & $10.1186 /$ bcr-1999-66594 \\
\hline \hline ArticleCitationID & $:$ & 66594 \\
\hline \hline ArticleSequenceNumber & $:$ & 36 \\
\hline \hline ArticleCategory & $:$ & Paper Report \\
\hline \hline ArticleFirstPage & $:$ & 1 \\
\hline \hline ArticleLastPage & $:$ & 4 \\
\hline \hline & $:$ & RegistrationDate : 1999-7-23 \\
\hline ArticleHistory & $:$ & OnlineDate \\
\hline \hline ArticleCopyright & $:$ & Current Science Ltd1999-7-23 \\
\hline \hline ArticleGrants & $:$ & \\
\hline \hline ArticleContext & $:$ & 1305811 \\
\hline \hline
\end{tabular}




\section{Keywords}

micro-metastases, prognosis, recurrence, stage, survival

\section{Introduction}

Although the widely used tumour-nodes-metastasis (TNM) staging system is generally regarded as an effective prognostic index for breast cancer, the search continues for other factors that might accurately predict which patients are at a higher risk of disease recurrence, metastasis and death. These patients may be most likely to benefit from adjuvant therapy and sound prognostic factors are therefore of immediate clinical relevance. Previous studies have suggested that the presence or absence of bone marrow micrometastasis may provide important independent prognostic information regarding diseasefree and overall survival with breast cancer.

\section{Aims}

This study describes the long-term follow-up of a group of women with primary breast cancer who had bone marrow aspirates taken at the time of initial surgery.

\section{Comments}

This study reiterates the importance of the traditional prognostic factors in predicting relapse-free and overall survival in women with primary breast cancer. With a median of 12.5 years of follow-up, data on bone marrow micrometastases did not provide any additional prognostic information once TNM stage had been taken into account.

\section{Methods}


From July 1981 to August 1986, 350 consecutive women with primary breast cancer were recruited from the breast units of three London Hospitals. Study participants ranged in age from 26 to 85 years, with a median age of 59 years. Each participant had bone marrow taken from eight sites prior to initial surgery. The eight marrow samples were pooled then examined immunocytochemically with antibody to epithelial membrane antigen (EMA). Adjuvant therapy was given to 141 patients: 95 patients received endocrine therapy and 35 received combination chemotherapy. Following surgery, patients were initially reviewed every 3 months and underwent clinical examination, blood tests (including liver function tests) and chest radiography. Bone scans were conducted every 6 months for 3 years, then annually thereafter. Most patients were discharged 5 years after surgery and follow-up continued through hospital records and contact with family physicians. All patients' records were flagged though the National Health Service Central Register for mortality status, and death certificates were reviewed for cause of death. Patients' relapse and survival was followed until January 1996. Seven women were lost to follow-up for recurrence status and two women were lost to follow-up for mortality. The association between micrometastases and various prognostic factors was investigated, as well as the effect of micrometastases on relapse-free and overall survival.

\section{Results}

Bone marrow micrometastasis were present in 89 (25\%) of the 350 women in the study. The presence of micrometastases was significantly associated with the adverse prognostic factors of increasing tumour size, presence of involved lymph nodes and vascular invasion. No association was seen with oestrogen receptor status or menopausal status. After a median follow-up of 12.5 years, $151(43 \%)$ patients had metastatic disease and $136(39 \%)$ had died from breast cancer. Crude 10-year relapse-free and overall survival were $43.9 \%$ (95\% CI 33.4-54.7) and 44.9\% (34.2-55.9) in women with micrometastases, compared to $62.7 \%$ (56.5-68.6) and 65.7\% (59.4-71.5) in women without micrometastases at baseline (P $<0.001)$. However, once tumour size, lymph node status and vascular invasion were taken into account, the effect of micrometastases on relapse-free and overall survival was no longer significant, with adjusted hazard ratios of 1.09 (0.74-1.61) and 1.21 (0.84-1.75), respectively.

\section{Discussion}

Although the presence of micrometastases at the time of initial surgery was associated with diminished relapse-free and overall survival, this prognostic relationship did not persist once tumour size, lymph node status and vascular invasion had been taken into account. This indicates that, in this dataset, micrometastatic status was not an independent predictor of recurrence or survival. This is in contrast to some previous studies with shorter follow-up (up to 36 months), which have found that micrometastases independently predicts outcome. It is possible that micrometastases may be a better predictor of relapse-free survival and overall survival in the years immediately after diagnosis but the importance may diminish over time. However, findings regarding the time dependency of the effect of micrometastases were non-significant in this study. The presence or absence of micrometastases at the time of surgery should not be used to guide the use of adjuvant therapy, as tumour size and lymph node 
status would be known in these women. Ascertainment of micrometastatic status using antibody to EMA is not necessarily the best method, and future work using techniques such as PCR or different monoclonal antibodies would be of interest.

\section{References}

1. Mansi JL, Gogas H, Bliss JM, Gazet J-C, Berger U, Coombes RC: Outcome of primary breast cancer patients with micrometastases: a long-term follow-up study. Lancet. 1999, 354: 197-202. 